\title{
NARRATIVAS, PRÁCTICAS REFLEXIVAS Y SABERES PEDAGÓGICOS EN DOCENTES EN FORMACIÓN
}

\section{NARRATIVES, REFLECTIVE PRACTICES AND PEDAGOGICAL KNOWLEDGE IN TEACHERS IN TRAINING}

\author{
Alejandra Nocetti de la Barra \\ Julio Hizmeri Fernández \\ Jocelyn Arriagada Candia
}

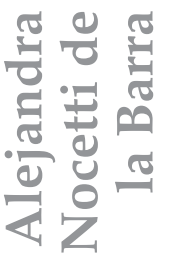

Doctora en Educación y Sociedad. Jefa programa Magister en Ciencias de la Educación, Universidad Católica de la Santísima Concepción, Chile. Miembro de REDFORMA (Red de Formadores de Formadores) y miembro de PIPR (Plataforma Internacional de Práctica Reflexiva).

anocetti@ucsc.cl

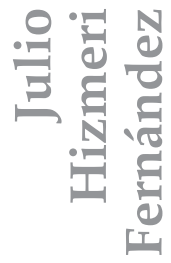

Doctor en Educación y Sociedad. Jefe Unidad de Práctica Pedagógica, Universidad Católica de la Santísima Concepción, Chile.

jhizmeri@ucsc.cl

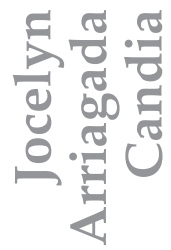

Candidata magister en Ciencias de la Educación, Universidad Católica de la Santísima Concepción, Chile. jarriagada@magisteredu.ucsc.cl 


\section{RESUMEN}

Chile mantiene el desafío de formar docentes reflexivos y, en ese contexto, el objetivo del estudio fue describir la experiencia con relatos sobre lo vivido, en los centros de prácticas, y analizar su relación con la práctica reflexiva y la construcción de saber pedagógico en estudiantes de tres programas de formación pedagógica, en la región de Biobío al sur de Chile. La investigación fue interpretativa con un diseño de casos múltiples. El estudiantado escribió relatos durante la práctica y los compartieron en un sitio virtual. Los resultados indican que escribir relatos significó «re-vivir, re-conocerse y re-flexionar» y al compartirlos, se «enriquecen», se «reconocen» y se «reconstruyen», a partir del saber pedagógico adquirido en la práctica docente. En conclusión, la narrativa estimula la práctica reflexiva y promueve procesos de formación y transformación del docente, a partir de una epistemología que se distancia de un modelo aplicacionista de la formación del profesorado.

Palabras clave: práctica reflexiva, saberes pedagógicos, narrativa, práctica docente.

\section{ABSTRACT}

Chile maintains the challenge of training reflective teachers and in this context, the objective of the study was to describe through stories about teachers' experiences in the practice centers analyzing their relationship with reflective practice and the construction of pedagogical knowledge. The study was conducted with students in three programs of pedagogical training in the Biobío region in southern Chile. The research was interpretive with multiple case design. The students wrote stories during the practice and shared them in a virtual site. The results indicate that writing stories meant «re-living, re-thinking and re-flexing» and by sharing them, they are «enriched», «recognized» and «reconstructed» from the pedagogical knowledge acquired in the teaching practice. In conclusion, the narrative stimulates reflective practice and promotes processes of teacher training and transformation, based on an epistemology that distance themselves from an applications model of teacher education. 
Keyword: Reflective Practice, Pedagogical Knowledge, Narrative, Teaching Practice.

\section{INTRODUCCIÓN}

Hoy se reconoce la importancia de la reflexión durante la formación de los profesionales (Schön, 1992), especialmente, porque significa una mirada epistemológica que se distancia de la postura técnica de la formación profesional. En el campo educativo, varios autores (Altet, 2010; Barcena, 2005; Perrenoud, 2004, Tardif, 2004) señalan que, poseer reflexión temprana, robustece el aprendizaje desde la experiencia misma (Domingo y Gómez 2014) y constituye el sustrato para la construcción de saber profesional que forma y transforma al futuro docente (Contreras, 2010; Tardif, 2004). Coherente con lo anterior, la política pública chilena — en materia de educación- evidencia la adhesión a estos planteamientos en los Estándares para la Formación Inicial Docente (2012), el Marco para la Buena Enseñanza (2003) y otras orientaciones para docentes que inician la carrera profesional.

Dada la relación entre práctica reflexiva y desarrollo profesional (Correa, 2011), podría afirmarse que esta constituye el motor de la práctica, porque se vincula con la construcción de saberes profesionales (Guevara, 2018; Labra y Fuentealba, 2011; Perrenoud, 2004; Tardif, 2004). En nuestro caso, nos adherimos a la propuesta de Contreras y Pérez de Lara (2010), quien establece que la experiencia, al ser analizada de modo reflexivo, moviliza la construcción de saberes —en este caso formativos - que articulan lo que aporta el sujeto desde su propia biografía escolar, lo que suscribe el proceso formativo y lo que le ocurre en términos profesionales, tanto en la institución universitaria como en la propia escuela. Todos estos elementos, al ser objeto de reflexión, poseen un alto potencial de aprendizaje para el futuro profesor.

Así, existen numerosas investigaciones sobre los recursos y dispositivos que estimularían una práctica reflexiva temprana durante la formación profesional. Destacan entre ellos, el de video grabación (Anijovich y Cappelletti, 2014; Davini, 2015; Husu, Toom y Patrikainen, 2008; Paquay y Wagner, 2010), el diario docente (Anijovich y 
Cappelletti, 2014; Caporossi, 2012; Costa, Ornique y Peire, 2014; Jarpa, Hass y Collao, 2017; Zabalza, 2004, Zeichner y Liston, 1999), el portafolio electrónico (Rodrígues y Rodríguez-Illera, 2014; Seckel y Font, 2016) y, más recientemente, el empleo de relatos de experiencias (Contreras, 2010; Suarez, 2007)

A nivel institucional, se trabaja en un modelo de formación práctica reflexiva que valora la experiencia como sustrato para construir saber pedagógico. En dicho contexto, el objetivo de esta investigación es describir la experiencia con relatos de experiencias vividas en los centros de prácticas por estudiantes de Pedagogía, y analizar su vinculación con la práctica reflexiva y la construcción de saber pedagógico en docentes en formación, de tres programas de formación de maestros en una universidad de la región de Biobío, al sur de Chile.

\section{ANTECEDENTES TEÓRICOS CONCEPTUALES \\ La Investigación Narrativa en Ciencias Sociales y en Educación}

El giro hermenéutico y narrativo abre la senda para entender los fenómenos sociales y educativos como textos, cuyo significado viene dado por la auto-interpretación que de ella dan los sujetos. De esta manera, se cree en la importancia de hacer emerger al sujeto y propiciar la reflexividad. Probablemente este sea uno de los aportes más relevantes de la creciente investigación biográfica y narrativa que la diferencia de otras perspectivas dentro de la orientación interpretativa.

El interés actual por los relatos y narrativa puede ser visto como la expresión de una actitud crítica hacia el conocimiento, como racionalidad técnica y formalismo científico. Todo lo cual expresa, por lo tanto, el deseo de describir e interpretar las experiencias significativas de la vida diaria en la escuela — desde la voz de sus protagonistas-, no como un rechazo de la ciencia, sino más bien como método para tratar las preocupaciones que normalmente quedan excluidas de la ciencia habitual. Por ello, como reflejo de este giro en las ciencias sociales, Santamaría y Marinas (1995) señalan que el fenómeno conforma 
un síntoma de época al que llaman el sintoma biográfico. Asimismo, Denzin (2003) señala que vivimos en el momento de la narración, añadiendo que todo lo estudiado está dentro de una representación narrativa o relato $\mathrm{y}$, por consiguiente, el mundo académico llama teorías a esas historias. Esto explicaría la gran cantidad de documentos personales y la multiplicidad de usos que está teniendo, desde hace décadas, extendiéndose y cubriendo diferentes disciplinas con objetivos distintos (Plummer, 1989).

El estudio de la narrativa, por lo tanto, es el estudio de la forma en que los seres humanos experimentamos el mundo. De esta idea general se deriva la tesis de que la educación es la construcción y la re-construcción personales y sociales; tanto los profesores como los alumnos son contadores de historias y también, personajes en las historias de los demás y en las suyas propias (pp. 11-12).

Cabe señalar que la investigación narrativa reclama un ideal de racionalidad post-clásico, superador del contraste establecido entre objetividad y subjetividad, para basarse en las evidencias originarias del mundo de la vida, desde los relatos de experiencia vivida. Por lo tanto, el relato de experiencia vivida capta la riqueza y los detalles de los significados en los asuntos humanos y educativos que no pueden expresarse en definiciones, enunciados factuales o proposiciones abstractas, ya que tiene cierto carácter de inefabilidad, como lo expresara Van Manen (2003). Por ello se trabajará con relatos de experiencias de los sujetos que dan cuenta de lo vivido.

Señalado esto, la experiencia y su relato constituyen elementos fundantes en la aproximación fenomenológica y hermenéutica, incorporando la dimensión temporal y, con ello, el reconocimiento histórico de la experiencia relacional en el seno de una comunidad lingüísticamente articulada. Así, no es de sorprender que Connelly y Clandinin (1995) enfaticen que los seres humanos somos organismos contadores de historia, organismos que, individual y socialmente, vivimos vidas relatadas. La narrativa dice relación tanto con la cualidad de la experiencia, vista como relato, como con las formas de construir sentido, a partir de hechos o acontecimientos temporales personales. 


\section{EL SABER PEDAGÓGICO Y EL SABER DE LA EXPERIENCIA}

El saber no se reduce a la ciencia, ni siquiera al conocimiento. El conocimiento sería el conjunto de los enunciados que describen objetos susceptibles de ser declarados verdaderos o falsos, con exclusión de todos los demás enunciados; mientras que la ciencia sería un subconjunto de conocimientos, construida con enunciados denotativos, y que impondría dos condiciones suplementarias para su aceptabilidad dentro del lenguaje considerado como pertinente por los expertos (Lyotard, 1984). Por su parte, el saber pedagógico se compone de dos perspectivas del saber; el saber pedagógico tiene que ver tanto con el saber de la experiencia práctica, como con el poso de sabiduría que va conformando nuestra experiencia, y que orienta el pensar y el vivir de lo educativo.

Se trata, por tanto, de un saber encarnado, que no es simplemente algo que se posee, sino parte de lo que se es, de tal manera que ser y saber no están escindidos (Contreras y Pérez de Lara, 2010). Así, el saber pedagógico es tal porque ha ido cuajando en la relación de interrogación de lo vivido, y en la mirada abierta a aquellos con quienes entramos en relación. En otras palabras, un saber que hace de la vivencia una experiencia con valor pedagógico y un saber en relación, atento a la solicitud pedagógica y al tacto pedagógico, empleando nociones de Van Manen (1998).

Dicho esto, el saber de la experiencia no se conforma por una simple interiorización de las formas habituales de comportamiento y su automatismo, sino que emerge del vivir y del actuar, manteniendo una relación pensante con el acontecer de las cosas, que interrumpe lo ya sabido y obliga de nuevo a mirar y pensar. Un saber que interpela a descentrarse de sí y que aprende a abrirse a lo inesperado y, por lo tanto, que se pregunta por el sentido de lo que pasa, tanto como su propio actuar, buscando más allá de un pensamiento fijo (Contreras y Pérez de Lara, 2010). Es un saber que se autoriza a aparecer —más allá de los parámetros y las prescripciones de perfiles de desarrollo docente (como las establecidas en el Marco para la Buena Enseñanza o las establecidas en los planes de formación) - y, por tanto, un saber en primera persona, que nace del acto inaugural de dar cuenta de la experiencia. O sea, un saber que constituye un acto poético y, por ende, fundacional que puede emerger al momento de relatar la experiencia vivida en educación. Esto 
porque muchos de los relatos contienen un saber que circula en el mundo exterior. Pues, «en muchos casos, los relatos no solo contienen un saber, sino que son en sí mismo el saber» (Jackson 1998, p. 28).

\section{METODOLOGÍA}

La investigación fue desarrollada con un enfoque interpretativo (Álvarez-Gayou, 2013; Crotty, 1988; Sandin, 2003; Van Manen, 2003), focalizándose en la experiencia de profesores en formación que emplean relatos en la asignatura de práctica pedagógica para adquirir saber, desde la experiencia, en el centro de práctica. Se empleó el método de estudio de casos intrínseco (Stake, 2013), el que tuvo un carácter descriptivo e interpretativo de la experiencia del estudiantado de las carreras de: Pedagogía en Educación Básica y Pedagogía en Educación Media en Biología y Matemática, tal como puede apreciarse en la siguiente tabla:

\section{Tabla 1. Caracterización de los casos.}

\begin{tabular}{|c|c|c|c|}
\hline & & $\mathbf{N}$ & $\%$ \\
\hline \multirow{3}{*}{$\begin{array}{l}\text { Programa } \\
\text { de formación }\end{array}$} & $\begin{array}{l}\text { Pedagogía en } \\
\text { educación } \\
\text { básica. }\end{array}$ & 6 & 23 \\
\hline & $\begin{array}{c}\text { Pedagogía en } \\
\text { educación media en } \\
\text { matemática. }\end{array}$ & 10 & 38,5 \\
\hline & $\begin{array}{c}\text { Pedagogía en } \\
\text { educación media en } \\
\text { biología. }\end{array}$ & 10 & 38,5 \\
\hline \multirow[t]{3}{*}{ Tipo práctica } & Práctica pedagógica. & 16 & 61,5 \\
\hline & Práctica profesional. & 10 & 38,5 \\
\hline & Total & 26 & 100 \\
\hline
\end{tabular}

Fuente: Elaboración propia sobre 26 casos válidos. 
De acuerdo con Flick (2015), la selección de los casos obedeció a dos criterios. En primer lugar, su potencial de transferibilidad; es decir, los programas seleccionados comparten características con otros planes de formación de universidades regionales del sur de Chile. En segundo lugar, se eligieron los casos por el acceso al campo (Vieytes, 2004); es decir, se disponía de apoyo institucional para trabajar con estos estudiantes de Pedagogía. Cabe indicar que se colaboró con un total de 26 docentes en formación, de los tres programas indicados anteriormente.

\section{Recogida de los Datos}

Se utilizó como estrategia de recogida de datos, la entrevista semiestructurada (Kvale, 2012) y la escritura de relatos de experiencia (Connelly y Clandinin, 1995; Bolívar, Segovia y Fernández-Cruz, 2001; Suárez, 2007). En esta investigación, las narrativas fueron parte de los trabajos desarrollados en el taller universitario y las preguntas que guiaron el relato fueron las siguientes: 1) ¿Qué suceso puntual, vivido en el centro de práctica, ha sido el más significativo durante este mes? 2) ¿Por qué fue significativa para ti dicha situación? ¿En qué sentido lo ocurrido transforma tu mirada del ejercicio de la profesión?

\section{Análisis de los Datos}

Se desarrolló un «análisis temático» a través de la reflexión macro-temática o aproximación holística y sentenciosa; la reflexión micro-temática a través de la aproximación selectiva o de marcaje y, finalmente, la aproximación detallada para obtener un conjunto de frases que «capturaran» los significados esenciales de la experiencia) (Van Manen, 2003). A partir de ello se desarrolló la obtención de resultados y conclusiones (Rodríguez, Gil y García, 1999). 


\section{Resultados}

A partir del análisis temático de los relatos, emergen dos dimensiones, por un lado, la experiencia de relatos, por otro, la experiencia de circulación de relatos, donde la primera se corresponde con una perspectiva individual y la segunda con una perspectiva entre pares.

\section{DIMENSIÓN TEMÁTICA: LA EXPERIENCIA DE ESCRITURA DE RELATOS}

A partir de la dimensión temática «La experiencia de escritura de relatos» se desprendieron tres focos de reflexión o planos: re-vivir, re-conocerse y re-flexionar.

\section{Re-Vivir}

El estudiantado reconoce que la escritura de relatos en la asignatura de práctica permitió «volver a vivir la experiencia», para extraer de ella ciertos saberes que transformaron el sentido que dan a la docencia, tal como lo ilustra en su relato una futura profesora de educación básica y Biología:

El hecho de pensar en una vivencia en el aula para relatarla y escribirla hace [que] nuevamente vivamos lo ocurrido, detalles que quizás en el momento no les dimos la importancia necesaria, pero que al recordarlos nos dice mucho más y uno va obteniendo un conocimiento de lo que nos pasó en el aula o en la escuela. P60: Narrativa-Básica.docx- 60:9.

Cuando uno se sienta y vuelve a repasar la situación para escribirla se hace muy significativo, ya que vuelves a vivir lo que pasó en ese momento, y uno va aprendiendo de esa experiencia; es como lo que a uno le queda de lo que le pasó y en ese nuevo conocimiento va cambiando el modo de ver la profesión. P24: Narrativa Biología-2017.docx - 24:3.

Por una parte, el «re-pensar» significó analizar detenidamente, y por otra, implicó pensar detalladamente. En primer lugar, reconocieron 
que habitualmente piensan rápidamente sobre lo que acontece en la clase, especialmente cuando enfrentan problemas o situaciones emergentes, siendo la actividad mental rápida, constante y gobernada por la urgencia de una respuesta a lo que acontece. En cambio, relatar significó pensar detenidamente sobre lo que sucede a diario, reflexionar sobre aquello y a partir de esa experiencia, replantearse la acción profesional. Lo expresado queda manifiesto a continuación:

Pensar sobre algo que está pasando en el momento es algo que se debe hacer de modo rápido, ya sea para resolver alguna situación problemática o emplear lo [que] emerge en favor del objetivo de la clase. Bajo esas circunstancias se requiere actuar de manera rápida, en cambio pensar con relatos significó pensar de modo más calmado o reposado. Eso te lleva a desarrollar una reflexión sobre lo vivido y de ese modo, una va aprendiendo de lo que nos pasa y, también, te lleva a replantearte la actuación docente para enfrentar de otro modo situaciones similares futuras. P64: Narrativa-Básica.docx - 64:2.

El pensar detenidamente los liberó de la urgencia de responder/ actuar de inmediato como una reacción ante situación profesional y, de acuerdo con los docentes en formación, los encauzó a extraer un conocimiento para enfrentar situaciones equivalentes, teniendo la escritura de relatos un alto potencial transformador de la práctica docente, tal como se visualiza en una reflexión de un estudiante de la carrera de Biología:

Escribir relatos me llevó a desarrollar un pensamiento apaciguado, pensar calmadamente en la solución a un problema, de modo que luego podía usar ese conocimiento en situaciones futuras similares y esa experiencia de poco fue transformando mi actuación profesional. P64: Narrativa-Biología. docx -64 .

En segundo lugar, los profesores en formación percibieron que el pensar detenidamente estimula en ellos la habilidad para pensar detalladamente. Ellos enfatizan que se trata de una auto observación intencional que diversifica los puntos de atención. Por consiguiente, afloran los actores, sus dichos y entredichos, reacciones y acciones. Todo lo cual se transforma en sustrato a partir del cual se va extrayendo un saber 
que emerge de la reflexión sobre lo vivido en una relación pensante con lo que le pasa y ocurre dentro o fuera del aula en un espacio educativo, lo que se refleja en la siguiente declaración:

Escribir relatos en la asignatura de práctica de modo habitual, desarrolló en mí la habilidad para poner atención en los detalles. Se trata de una auto observación intencional; aparecen los diferentes actores implicados, las acciones se analizan y se piensa en las consecuencias de lo ocurrido. Luego, a partir de una lectura exhaustiva de lo relatado, se puede ir rescatando aquella información que resulta importante para mejorar la práctica pedagógica y, entonces, esta reflexión va incrementando el conocimiento de la profesión. P25: Narrativa-Biología.docx - 25:5.

\section{Re-Conocerse}

Un gran número de profesores en formación percibe que la escritura de relatos permite un nuevo conocimiento de sí: examinar lo que se piensa y siente sobre la profesión docente. Esto significa volver hacia sí mismo para re-conocerse como profesor; dicho conocimiento les permite ir desarrollando identidad docente. Lo anterior se visualiza en la siguiente experiencia:

Fue un buen ejercicio de reflexión trabajar a través de la escritura de nuestros relatos. Y quiero destacar que, a través de este relato, pude ordenar ciertas ideas sobre la docencia: reconocer lo que yo siento como docente. Al comprender un poco mejor cuáles son mis ideas y desafíos con esta profesión, uno se va conociendo como profesor. Es como otra dimensión de uno mismo; se trata de ir reconociéndose como profesor. P59: Narrativa-Matemática.docx - 59:5.

Asimismo, la mayoría del estudiando tiende a vincular el re-conocerse con una autoevaluación de la propia acción profesional. En esa perspectiva, manifestaron que relatar experiencias les ayudaba a identificar debilidades y también, a plantearse el modo de superar aquellas deficiencias, especialmente en el caso de estudiantes que trabajaron de modo complementario con análisis de videos de clases, tal como se plantea a continuación: 
Fui recordando las vivencias en el establecimiento mediante las observaciones que realicé de mi clase filmada. Los relatos me permitieron encontrar aquella debilidad que pasa desapercibida y me motivó a mejorar para una próxima clase, ya sea reforzando una competencia en los estudiantes o mejorando mi propia competencia docente. Verme en el video me hizo reflexionar y luego, [al] escribir sobre aquello, definitivamente me comprometió con un mejoramiento de mi práctica. P25: Narrativa-Biología.docx - 25:14.

Por otra parte, experimentaron que los errores pueden transformarse en aprendizaje profesional a partir de su análisis reflexivo:

Logré a través de los relatos y también, a partir de la maduración con la cual uno termina esta carrera, ver de manera positiva los errores y no quedarse solo con lo negativo, sino que rescatar algo de ello y cambiarlo. En ese sentido, creo que no basta con identificar y reflexionar en torno a los aspectos deficientes, sino que hay que trabajar en el mejoramiento de la propia práctica docente y no sé si con los relatos se llega a eso. P24: Narrativa-Biología.docx - 24:7.

Se agrega a la inquietud anterior, cierto cuestionamiento profesional, es decir, hasta qué punto se cumple con lo que se espera de un profesional de la educación. En ese sentido, existe temor de no responder a la expectativa profesional; ello se minimiza cuando se comparten experiencias similares y se comienza a comprender que dicho cuestionamiento es parte del proceso de transformarse en profesional de la educación.

Relatar supone re-conocerse y juzgar el trabajo desarrollado en el aula. Muchas veces en esta experiencia, reflexioné acerca de si respondería a las expectativas que tienen los estudiantes acerca de mi labor dentro de la sala de clases y me atemoricé, pero al compartir los relatos con otras compañeras, bajó mi ansiedad, porque a la mayoría le pasa lo mismo. P62: Narrativa-Básica.docx - 62:8. 


\section{Re-Flexionar}

El flexionarse o volverse a sí mismo, fue percibido por la mayoría del estudiantado en sus relatos de experiencias. En este sentido, el pensar detenida y detalladamente, y reconocerse a través del relato, permite a los docentes en formación contar con un tiempo destinado a la reflexión pedagógica, cuestión que valoran y que declaran como algo poco común en experiencias anteriores. Así lo escribe una profesora en formación de la carrera de Biología y Matemática:

Es totalmente diferente a la experiencia en otras prácticas, porque se genera una nueva perspectiva, con mucho más detalle, de lo que nos ocurrió. El pensamiento es más calmado y predispone al análisis reflexivo de lo vivido y eso es valioso para nosotras. P25: Narrativa Matemática-2017.docx - 25:4.

En cuanto al proceso de escribir relatos, debo decir que si bien a mí no me gusta leer y tampoco escribir, encuentro que es un proceso valioso hacer relatos porque obliga a pensar y recordar lo vivido, y ese es el motor básico para reflexionar sobre nuestras acciones y de a poco ir mejorando, cuestión que no vemos de modo sistemático en las prácticas anteriores. P26: Narrativa-Biología.docx - 26:3.

Se evidencia que la reflexión desprendida de la escritura de relatos deja de ser episódica y, por consiguiente, estimula una experiencia de práctica reflexiva temprana, que los docentes en formación entienden como parte del desarrollo profesional. Se percibe y valora la construcción de saberes que emergen de la relación pensante con la propia experiencia, en contextos de práctica en los establecimientos educacionales, re-significando el valor que tiene la formación práctica en el plan de formación, lo que queda de manifiesto a continuación:

Pensar sobre nuestro actuar, mediante relatos, transforma la reflexión en una costumbre y se hace un hábito que estimula nuestro desarrollo profesional; adquirimos conocimientos a partir de las propias experiencias, no experiencias que nos da otra persona, sino lo vivido por uno y eso constituye un aprendizaje profundo de la profesión. P26: NarrativaBiología.docx - 26:4. 
Sin duda, el hecho de escribir sobre las vivencias ocurridas durante la estadía en nuestros centros de práctica, ayudó a reflexionar de modo profundo porque una va adquiriendo conocimiento de aquello que va viviendo y entonces, uno se desarrolla profesionalmente. P64: Narrativa Básica-docx - 64:1.

Hay dos modos en que el relato, a juicio del estudiantado, estimula el mejoramiento de la propia práctica. Por una parte, acentúa la reflexión sobre la acción docente, particularmente incrementando la problematización sobre la actuación docente y el planteamiento de dilemas. Por otra, el relato aumenta la vinculación pedagógica entre docente y estudiantado, ya que los estudiantes siempre están presentes de modo explícito o implícito en las situaciones que narran los profesores en formación:

Entonces, al escribir el relato, reforzamos la reflexión, porque pensamos sobre nuestro actuar después de la clase y nos planteamos problemas desapercibidos anteriormente. Igualmente, aumentan los dilemas cuando pensamos más consciente[mente] en el estudiantado, y entonces uno duda y entonces sirve para cambiar o reforzar nuestra actuación. P26: Narrativa-Biología.docx - 28:1.

Con cada una de las vivencias dentro del aula que trabajé con los relatos, me volví más reflexiva. Además, me interesé más por conocer la vida de los estudiantes; averigüé qué ocurría con algunos niños y niñas, y esto aumentó mi vínculo con ellos. Yo creo que esa estrecha relación con ellos [y] afectó positivamente mi reflexión y comencé a reflexionar más sobre lo que hacía en la clase. P64: Narrativa-Básica.docx - 64:6.

\section{DIMENSIÓN TEMÁTICA: LA CIRCULACIÓN DE RELATOS}

En este estudio se emplea la noción de «circulación de relatos» como la disposición de estos en una plataforma en que todos los participantes puedan acceder a ellos; particularmente, una vez al mes, tuvieron que seleccionar una de las narrativas (relatos) y escribir qué les ocurría 
a ellos con esa experiencia. En el contexto de esta experiencia se configuraron tres focos de reflexión o planos: 1) Enriquecerse con otras experiencias. 2) Re-conocerse en la vivencia del otro. 3) Reconstruirse desde el saber pedagógico.

\section{Enriquecerse con Experiencias de Otro}

Se observó que el estudiantado valora compartir las vivencias de los centros educacionales, porque reconocen que falta trabajo colaborativo en las escuelas y liceos, ya que el trabajo del docente se concibe como una actuación solitaria; en ese sentido, esta experiencia los lleva a cambiar el modo de ver la profesión: menos individual y más colectiva.

Yo pensaba que la educación es algo que se debe trabajar de manera individual, para ser capaz de ver tus propios errores, pero con esta experiencia con los relatos compartidos también pienso que hay que intercambiar vivencias. Muchas veces en los colegios los problemas se trabajan aislados, creo que es importante comunicar lo que nos pasa y así trabajar en conjunto en opciones de mejoramiento. En ese sentido, pensar sobre lo vivido con relatos se hace significativo, especialmente para cambiar el modo de ver la profesión más como [trabajo] colectivo y no como algo individual. P24: Narrativa-Matemática-2017.docx - 24:7.

La mayoría precisó que en la medida que existe más análisis de las problemáticas entre pares, se incrementan las soluciones posibles, se enriquece la comprensión de las causas y de los efectos de una acción profesional particular. Entonces, uno se abre a nuevos puntos de vistas y se nos ocurren más opciones para hacer frente a las dificultades propias de nuestra docencia. Al respecto, una docente en formación, del programa de Pedagogía de Educación Básica, plantea lo siguiente:

Escribir relatos para compartirlos con otras compañeras, [es] tal vez una de las cuestiones más valiosas de esta experiencia, porque uno puede conocer una visión diferente del problema y también, [es] otro modo de dar soluciones o ayudas. Esto nos enriqueció mucho porque ya no se trata de lo que uno aprende por sí mismo, sino también, con la visión de otras compañeras. P64: Narrativa-Básica.docx - 64:3. 
Además como compartimos experiencias y relatos con otros compañeros, más de una de esas experiencias causó un impacto en mí y me ayudó a comprender, desde el punto de vista de otro compañero, cómo reaccionar ante aquellas situaciones. P60: Narrativa-Básica.docx - 60:14.

Por otra parte, percibieron que el compartir sus relatos de experiencias vividas, con compañeros de otros programas de formación, amplía la comprensión de la profesión docente, diversifican el conocimiento sobre «la docencia», y se reconocen problemas comunes y específicos. Además, esto les significa la oportunidad de ir construyendo una identidad docente que sobrepasa los límites de las definiciones de la profesión, de acuerdo con la disciplina que se enseña.

La experiencia de compartir relatos de vivencias con nuestros pares, significó ampliar la comprensión de la docencia, ya que se accede a otras experiencias escolares, diferentes problemas de la enseñanza, algunos comunes y otros específicos de cada disciplina. Esto ayuda, de manera positiva, a desarrollar la capacidad de pensar críticamente y, además, desarrolla en nosotros una mirada de nuestra profesión que va más allá de la enseñanza de la propia disciplina; es decir, ante todo somos profesionales de la educación y esto no lo había percibido antes. P24: Narrativa- Biología.docx - 24:6.

\section{Re-Conocerse en la Experiencia del Otro}

De acuerdo con los estudiantes, compartir relatos de experiencia llevó, por una parte, a reconocer que existen experiencias comunes a los docentes en formación que están desarrollando su práctica pedagógica, independientemente de la disciplina que enseñen y esto disminuye la tensión, porque reconocen que obedece a la etapa de su formación:

Cuando leí los relatos de mis compañeros, reconocí en sus experiencias cuestiones que también me habían pasado a mí y entonces, uno se da cuenta que nos han pasado las mismas cosas o algo similar. Como que eso baja un poco la ansiedad, porque las problemáticas son comunes y por lo tanto, no se trata de un problema personal, sino [de] una cuestión 
de la profesión o de la etapa que estamos viviendo, supongo. P60: Narrativa-Matemática.docx - 60:10.

Además, la lectura de las experiencias del otro estimula la toma de conciencia de aspectos de la propia enseñanza que no habían sido reconocidos anteriormente durante la formación. Entonces, la experiencia del otro resuena en ellos y los hace más conscientes de su propia experiencia de profesor durante el desarrollo de sus prácticas pedagógicas en los centros educacionales. Al respecto, un estudiante de la carrera de Biología comenta su experiencia:

Pensar situaciones, escribirlas en forma de relato y compartirlas, hace que uno sea más consciente de lo que está pasando en su sala de clases y en su alrededor. Muchas veces me pasó que leer lo que le sucedía al otro, me llevaba a pensar en mi propia experiencia y, entonces, también mi propio conocimiento como profesor se fue enriqueciendo. P24: Narrativa-Biología.docx - 24:5.

\section{Reconstruirse desde el Saber Pedagógico}

El estudiantado percibe que va aprendiendo sobre el ejercicio de la profesión, porque los relatos se centran en situaciones que son significativas para ellos y en el análisis compartido, se va extrayendo conocimiento sobre la relación entre el docente y el estudiantado, la enseñanza de valores o la contención emocional y, en el caso de la educación media, se van preparando mejor para otras situaciones futuras.

Los relatos me permitieron, de alguna manera, establecer las vivencias más significativas dentro de aula; por ejemplo, contener al estudiante de enseñanza media es algo que se aprende en la experiencia. Al compartir las narrativas aumentó el conocimiento que se puede adquirir a partir de una situación particular y, de este modo, mejorar la práctica docente en situaciones similares futuras. P25: Narrativa-Biología.docx - 25:1.

Por otra parte, al compartir relatos, el estudiantado se hace más consciente que el conocimiento instrumental de la formación recibida en la universidad y las orientaciones para el trabajo que ofrece 
el Ministerio de Educación, no son suficientes para desarrollar una práctica docente pertinente $\mathrm{y}$, por lo tanto, se requiere de un saber que se va gestando en el análisis sistemático de la propia experiencia a nivel individual y grupal.

Compartir la reflexión de modo narrativo, me ha permitido aprender sobre la labor docente mediante el análisis de diferentes situaciones que son significativas para nosotros. Además, me ayudó a darme cuenta que hay muchas situaciones que se escapan de lo enseñado en la universidad y de lo que dictan los programas del Ministerio de Educación y, en ese sentido, hay que generar espacio para el análisis compartido de lo que nos ocurre y aprender entre todos. P27: Narrativa-Básica.docx - 27:7.

Se observa que hacer circular relatos refuerza la idea de que la construcción de saberes desde la experiencia, al compartirla con los compañeros, amplía el conocimiento sobre la profesión, develando aspectos pedagógicos no contemplados de forma individual:

Escribir mis propios relatos, compartir con los compañeros el conocimiento que voy adquiriendo, significó ampliar mi conocimiento sobre el ejercicio de la profesión. Me di cuenta que gran parte de nuestro trabajo no será solamente enseñar la matemática, lenguajes o biología, sino hacerse cargo de la formación de mis estudiantes. P27: NarrativaBiología2017.docx - 27:5.

Otro docente en formación agrega que no sabe si está preparado para asumir la tarea docente, pero ha comprendido que el ejercicio de la profesión se favorece por un saber pedagógico que surge desde lo particular y lo común, y que alimenta la vocación a la luz de lo verdaderamente significativo:

El compartir los relatos entre nosotros lo veo como una manera de «alimentación de la vocación», la cual va más allá de los siempre y cotidianos comentarios de la sala de profesores. Por el contrario, se trata de una reflexión sobre la vocación a partir de lo significativo que vivimos en los centros de práctica. A veces fueron cuestiones particulares y también, en muchas ocasiones, hay temas que son comunes a todos independientes de la carrera. P59: Narrativa-Básica.docx - 59:6. 


\section{DISCUSIÓN Y CONSIDERACIONES FINALES}

Los resultados del estudio indican que el empleo de relatos de experiencias en la asignatura de práctica pedagógica, estimula la práctica reflexiva en los profesores en formación. Cabe subrayar que la experiencia, a nivel individual, favoreció una reflexión sobre la acción significativa en el aula y significó «re-vivir, reconocerse». Se observa que en este proceso hay una vuelta a sí mismo, lo que hace presumir que se le otorga mayor valor a la propia experiencia como sustrato para la construcción de saber pedagógico. Lo anterior podría significar un avance hacia un modelo de formación práctico y situado que releva la importancia de los profesores en servicio en la formación del profesorado.

En la dimensión grupal del trabajo con los relatos, referido a la circulación de los relatos en la plataforma y la escritura de meta-relatos, basadas en las experiencias de los compañeros, significó «enriquecerse, reconocerse en el otro y reconstruirse a partir del saber pedagógico» que emerge del análisis reflexivo conjunto con pares. Esto denota dos cuestiones relevantes, por una parte, esta experiencia refuerza una identidad profesional que se co-construye a partir de la comprensión de la tarea profesional que se logra en conjunto y otorga un lugar al saber que se extrae desde el relato que explicita lo vivido en los centros escolares, reafirmando la idea del profesional de la educación como un sujeto de conocimiento, lo que evidentemente afectará su identidad profesional.

Además, la elaboración del relato siguiendo los tres pasos propuestos en este estudio, a saber: describir una situación significativa; luego, explicar por qué es significativa, y pensar cómo lo vivido transforma la perspectiva personal, demuestra la importancia de guiar el proceso de escritura desde un enfoque de práctica reflexiva. Asimismo, el hecho de dejar libre la elección de la situación a describir, develó que el contenido de la reflexión sobre lo que viven los profesores en formación, corresponde más con asuntos vinculados a la relación pedagógica y el manejo de la complejidad del aula, que con aspectos didácticos referidos a la enseñanza de un contenido disciplinar específico, como Lenguaje, Matemática o Ciencias Naturales. 
Los resultados reafirman lo que reporta la literatura en cuanto a la importancia de enseñar de modo intencional la reflexión docente (Domingo y Gómez, 2014; Perrenoud, 2004) y, en ese sentido, la experiencia con relatos de experiencias, refuerza el hábito de la práctica reflexiva en las asignaturas de práctica pedagógica (Costa, Ornique y Peire, 2014). Esto significa que no solo en los programas de formación se debe trabajar con relatos $\mathrm{u}$ otros dispositivos, sino que se debe guiar la experiencia reflexiva, asegurando cierta sistematicidad y el avance hacia una práctica reflexiva crítica (Fernández y Ramírez, 2015).

Asimismo, los resultados también evidencian la vinculación entre el empleo de relatos y la construcción de la identidad profesional (Madrid-Vivar y Mayorga-Fernández, 2015; Vera, 2010). Respecto de ello, este estudio evidencia que los compañeros co-construyen una visión de la profesión, a partir de los relatos compartidos en la plataforma. Esto resulta relevante, especialmente porque la identidad se asocia con el proceso de re-construcción desde el saber que emerge de la experiencia «revivida y repensada» (Contreras, 2013; Álvarez, Porta y Sarasa, 2010: Lozano, 2013).

Se observó que la mayoría relató experiencias sobre la relación pedagógica, la identificación de dificultades y la insuficiente preparación para abordar experiencias complejas en el aula. Así, gracias a los relatos y las experiencias de lectura se adentran en las vivencias y, a su luz, abordan la complejidad epistemológica del saber docente, ampliando el foco de atención hacia dimensiones más allá del plano de la didáctica del contenido.

Este hallazgo resulta relevante porque la formación inicial docente de cuño academicista descansa en reflexiones sobre el contenido y el enfoque de la enseñanza, evidenciándose un marcado enfoque técnico, como lo constatan Astudillo, Rivarosa y Ortiz (2014). Por el contrario, los participantes de esta experiencia con relatos toman consciencia de recibir una preparación instrumental en la universidad y que la realidad les exige competencias a desarrollar en la experiencia práctica, reiterando el valor de la formación práctica, especialmente cuando posee un enfoque práctico reflexivo. La formación bajo un enfoque aplicacionista suscita prácticas formativas inspiradas en un modelo ya obsoleto, de supuesta aplicación directa de la teoría a la práctica (Korthagen, 
2010), es decir, prácticas de enseñanza basadas en un modelo formativo deductivo que propician la distancia entre la formación inicial y la realidad escolar (Cochran-Smith y Lytle, 1999/2002).

En este sentido, la experiencia suscitó una reflexión en doble dirección porque no solo experimentaron la reflexión al escribir su propio relato, sino también al leer y comentar la experiencia del otro: la experiencia repensada, revivida enriquecida y reconstruida va formándoles y transformándoles.

Finalmente, las implicancias formativas se sitúan, al menos, en dos ámbitos. Por un lado, muestra una modalidad de trabajo con relatos de experiencias -enriquecidas con el análisis de compañeros-y que se constituyen en el sustrato para la construcción de saber pedagógico, distanciándose de un modelo de formación práctica «aplicacionista» para transitar hacia una propuesta formativa sustentada en un enfoque reflexivo. Y, por otro, en la relación escuela/universidad, surge el desafío de volcar estudios hacia las experiencias vividas en las escuelas y los saberes del profesorado, trayéndolos a la formación inicial docente.

\section{Agradecimiento}

Este proyecto de investigación (DINREG- 02/2018) fue apoyado por la Dirección de Investigación de la Universidad Católica de la Santísima Concepción, a la que agradecemos el financiamiento de nuestro estudio. 


\section{REFERENCIAS}

Altet, M. (2010). «La competencia del maestro profesional o la importancia de saber analizar las prácticas». En: Paquay, L., Altet, M., Charlier, E., Perrenoud, P. (2010). Formación profesional del maestro. Estrategias y Competencias (pp. 33-48). México: Fondo de Cultura Económica.

Álvarez-Gayou, J. (2013). Cómo hacer una investigación cualitativa. Fundamentos y metodología ( $2^{\circ}$ ed.). México: Paidós.

Álvarez, Z., Porta, L. y Sarasa, M. (2010). «Itinerarios de la buena enseñanza a partir de los relatos biográficos docentes». Profesorado. Revista de Currículum y Formación de Profesores, vol. 14 (3), 89-98.

Anijovich, A., Cappelletti, G. (2014). Las prácticas como eje de la formación docente. Argentina: Editorial Universitaria de Buenos Aires.

Astudillo, C., Rivarosa, A., y Ortiz, F. (2012). «Reflexión docente y diseño de secuencias didácticas en un contexto de formación de futuros profesores de ciencias naturales». Perspectiva Educacional, Vol. 53(1), 130-144.

Bárcena, F. (2005). La experiencia reflexiva en educación. Barcelona: Paidós.

Bolívar A., Segovia D., y Fernández-Cruz, M. (2001). La investigación biográfico-narrativa en educación. Enfoque y metodología. Madrid: Muralla.

Caporossi, A. (2012). «La narrativa como dispositivo para la construcción del conocimiento profesional de las prácticas docentes» (107-149). Sanjurjo, L. (Coord.), Caporossi, A., España, A., Alfonso, I. y Foresi, M. (2012). Los dispositivos para la formación en las prácticas profesionales. Argentina: Homo Sapiens.

Cochran-Smith, M. \& Lytle, S. L. (1999). «Relationships of knowledge and practice: Teacher learning in communities». Review of Research in Education, 24, 249-305.

Cochran-Smith, M. y Lytle, S. L. (2002). Dentro/Fuera. Enseñantes que investigan. Madrid: Akal. 
Connelly, F. y Clandinin, J. (1995). «Relatos de experiencia e investigación narrativa». En Larrosa, J. (ed), Déjame que te cuente. Ensayos sobre narrativa y educación. Barcelona: Laertes.

Contreras, J. (2010). «Ser y saber en la formación didáctica del profesorado: una visión personal». Revista Interuniversitaria de Profesorado, 24(2), 61-81. Recuperado de: http://www.redalyc.org/articulo. oa?id=27419198004

Contreras, J. y Pérez de Lara, N. (2010). Investigar la Experiencia Educativa. Madrid: Morata.

Contreras, J. (2013). «El saber de la experiencia en la formación inicial del profesorado». Revista Interuniversitaria de Profesorado, 27(3), 125136.

Correa, E. (2011). «La práctica docente: una oportunidad de desarrollo profesional». Revista Perspectiva Educacional, 50(2), 77-95

Crotty, M. (1998). The foundations of social research: Meaning and perspective in the research process. London; Thousand Oaks; New Dehli: Sage.

Costa, P., Ornique, M. y Peire, J. (2014). «Los Diarios Formativos». En: Anijovich y Cappelletti (2014). Las prácticas como eje de la formación docente. Buenos Aires: Eudeba.

Davini, M. (2015). La formación en la práctica docente. Argentina: Paidós.

Denzin, N. (2003). «Foreword: narrative's moment». In M. Andrews, S. Sclater, C. Squire \& A. Treacher (Eds.), Lines of narrative, (pp. 11-13). Londres: Routledge.

Domingo, A. y Gómez, M. (2014). La práctica reflexiva. Bases, modelos e instrumentos. Madrid: Narcea.

Farías, G. y Ramírez, M. (2007). «La formación reflexiva de los docentes mediante el desarrollo del portafolio electrónico de evidencias». IX Congreso Nacional Investigación Educativa, 5 al 9 de noviembre de 2007. 
Fernández, M. y Ramírez, P. (enero 2006). «Los relatos de experiencias escolares en la formación docente». Revista Iberoamericana de Educación, 37(4).

Flick, U. (2015). Diseño de la investigación cualitativa. Madrid: Ediciones Morata.

Guevara, J. (2018). «Saberes prácticos en la formación docente para la educación inicial». Pedagogía y Saberes, (48), 127-139.

Husu, J., Toom, A., y Patrikainen, S. (2008). «Guided reflection as a means to demonstrate and develop student teachers reflective competencies». Reflective Practice. Vol. 9, n. 1, 37-51.

Jackson, P. W. (1998). «Sobre el lugar de la narrativa en la enseñanza». En McEwan, H. y Egan, K. (comp.). La narrativa en la enseñanza, el aprendizaje y la investigación. Buenos Aires: Amorrortu editores.

Jarpa, M., Hass, V., y Collao, D. (2017). «Escritura para la reflexión pedagógica: rol y función del diario del profesor en formación en las Prácticas Iniciales». Estudios Pedagógicos, XLIII, n. 2, pp. 163-178.

Korthagen, F. (2001). Linking practice and theory. The pedagogy of realistic Teacher Education. Londres: LEA.

Korthagen, F. (2010). «La práctica, la teoría y la persona en la formación del profesorado». Revista Interuniversitaria de Formación del profesorado, 68, 83-102.

Kvale, S. (2011). Las entrevistas en investigación cualitativa. Madrid: Morata.

Labra, P. y Fuentealba, R. (2011). «Formación inicial docente: Una mirada al proceso de construcción de conocimiento profesional de desde la práctica». Foro Educacional, n. 19, 143-173.

Lozano, I. (2013). «La formación de la identidad del maestro, una construcción entre saber pedagógico y la investigación». Pedagogía y Saberes, (39), 99-106.

Lyotard, J. (1984). La condición postmoderna: Informe sobre el saber. Madrid: Cátedra. 
Madrid-Vivar, D. y Mayorga-Fernández, M. (2015). «Construcción y reconstrucción de la identidad de los futuros docentes a través de sus autobiografías académicas». Revista Complutense de Educación, 28(2), 375-389.

Ministerio de Educación (2003). Marco para la Buena Enseñanza. Santiago, Chile: CPEIP.

Ministerio de Educación (2012). Estándares orientadores para carreras de pedagogía en educación media. Santiago, Chile: Lom Ediciones.

Paquay L., y Wagner, M. (2010). «Formación continua y video formación: qué habilidades se deben priorizar». En: Paquay, L., Altet, M., Charlier, E., Perrenoud, P. (2010). La formación profesional del maestro. Estrategias y Competencias (pp. 222-263). México: Fondo de Cultura Económica.

Perrenoud, P. (2004). Desarrollar la práctica Reflexiva en el oficio de enseñar. Barcelona: Graó.

Plummer, K. (1989). Los documentos personales. Introducción a los problemas y bibliografía del método humanista. Madrid: Siglo XXI.

Rodríguez, G., Gil, J., García, E. (1999) Metodología de la Investigación Cualitativa. España: Aljibe.

Rodrígues, R. y Rodríguez-Illera, J.R. (2014). «El portafolio digital como soporte de la práctica reflexiva en la formación docente». Revista Iberoamericana de Educación, vol. 65, 53-74.

Sandin, M. (2003). Investigación cualitativa en educación. Fundamentos y tradiciones. Madrid: Mac-GrawHill.

Santamaría, C. y Marinas, J. M. (1995). «Historias de vida e historia oral». En Delgado, J.M. y Gutiérrez, J. (Eds.) Métodos y técnicas cualitativas de investigación en ciencias sociales. (pp. 257-285). Madrid: Síntesis.

Seckel, M. J. y Font, V. (2016). «El portafolio como herramienta para desarrollar y evaluar la competencia reflexiva en futuros profesores de matemática». En J. A. Macías, A. Jiménez, J. L. González, M. T. Sánchez, P. Hernández, C. Fernández, F. J. Ruiz, T. Fernández y A. Berciano (Eds.), Investigación en Educación Matemática XX (pp. 499508). Málaga: SEIEM. 
Schön, D. (1992). La formación de profesionales reflexivos. Hacia un nuevo diseño de la enseñanza y el aprendizaje en las profesiones. Barcelona: Paidós.

Stake, R. (2013). «Estudios de casos cualitativos». En Denzin, N. y Lincoln, Y. (Coords.), Las estrategias de investigación cualitativa (pp. 154-197). España: Gedisa.

Suárez, D. (2007). «Docentes, narrativa e investigación educativa. La documentación narrativa de las prácticas docentes y la indagación pedagógica del mundo y las experiencias escolares». En Sverdlick, I. (Comp.), La investigación educativa. Una herramienta de conocimiento y de acción. Buenos Aires: Novedades Educativas.

Tardif, M. (2004). Los saberes del docente y su desarrollo profesional. Madrid: Narcea.

Van Manen, M. (1998). El tacto de la enseñanza. Barcelona: Paidós.

Van Manen, M. (2010). El tacto en la enseñanza. Barcelona: Paidós.

Van Manen, M. (2003). Investigación educativa y experiencia vivida. Barcelona: Idea Books.

Vera, J. (2010). «Autobiografía, identidad docente y conocimiento didáctico en la enseñanza de la educación física». Revista de Educación, Vol. 21(2), 423-441.

Vieytes, R. (2004). Metodología de la investigación en organizaciones, mercado y sociedad. Argentina: Editorial de las Ciencias.

Zabalza, M. (2004). Diarios de clase. Un instrumento de investigación y desarrollo profesional. Madrid: Narcea.

Zeichner, K. y Listón, D. (1999). «Enseñar a reflexionar a los futuros docentes». En Pérez, Gómez, A. Desarrollo profesional docente: Política, investigación y práctica (pp. 506, 532). Madrid: Ed. AKAL. 